\title{
Sehnenverletzungen: Strecksehnendefektverletzungen im Bereich der Finger
}

\author{
Wiebke Petersen, Hans-Eberhard Schaller, Afshin Rahmanian-Schwarz
}

\section{Zusammenfassung}

Der Strecksehnenapparat der Finger hat einen anatomisch komplexen Aufbau und ist aufgrund seines oberflächlichen Verlaufs leicht anfällig für Verletzungen. Abhängig von dem Verletzungsmechanismus, der Komplexität und der Höhe der Verletzung gelten für die Behandlung von Strecksehnenverletzungen verschiedene therapeutische Richtlinien. Die Versorgung von Sehnendefektverletzungen bedarf meist komplexer mehrzeitiger Eingriffe und stellt eine besondere Herausforderung für den behandelnden Arzt dar. Dabei sind die genaue anatomische Kenntnis und ausreichend handchirurgische Erfahrung maßgeblich für die Qualität der Versorgung mit Erhalt der Feinmotorik und Koordination der Hand. Darüber hinaus kann nur ein intensives postoperatives Behandlungsmanagement mit adäquater Ruhigstellung und funktioneller Nachbehandlung den $\mathrm{Er}$ folg der Behandlung sichern.

\section{Tendon Injuries: Extensor Tendon Defect Injuries to the Fingers}

The extensor apparatus of the finger has an anatomically complex structure and is prone to injury due to its superficial course. Depending on the type of injury, the complexity and the level of injury, various therapeutic guidelines apply for the treatment of extensor tendon injuries. In particular, the treatment of tendon defect injuries often requires complex multiple interventions, which can pose a big challenge for the treating physician. Thereby the exact anatomic knowledge and sufficient hand surgical experience are important for high-quality care, preservation of range of motion and to avoid complications. Additionally only an intensive postoperative management with adequate immobilisation and functional rehabilitation can ensure the success of the treatment.

\section{Ätiologie}

Der Strecksehnenapparat der Finger hat einen anatomisch komplexen Aufbau und ist aufgrund seines oberflächlichen Verlaufs anfällig für Verletzungen. Neben Schnittwunden sind auch Biss-, Quetsch- oder Abrasionsverletzungen, Verbrennungen sowie Ausrissverletzungen ursächlich für Verletzungen. Sie werden häufig in der Notaufnahme diagnostiziert und nicht selten in ihrer Komplexität unterschätzt.

Gerade bei Kombinationsverletzungen mit Beteiligung mehrerer Strukturen oder bei Vorliegen von Sehnendefektver-

OP-JOURNAL 2015; 31: 72-77

(c) Georg Thieme Verlag KG Stuttgart · New York DOI http://dx.doi.org/10.1055/s-0035-1546205

letzungen sind der Zeitpunkt und das Prinzip der Versorgung maßgeblich für den Erhalt der Feinmotorik und Koordination der Finger.

Nur die genauen anatomischen Kenntnisse über den Aufbau und die Anatomie des Streckapparats sowie das Zusammenspiel verschiedener Sehnenanteile ermöglicht eine adäquate funktionelle Rekonstruktion nach Strecksehnenverletzungen mit einem langfristigen Erfolg.

\section{Anatomie}

Der Strecksehnenapparat besteht aus mehreren anatomischen Strukturen, einschließlich der langen Fingerstrecker (die extrinsische Muskulatur), kurzen Handmuskeln (die intrinsische Muskulatur) wie die Mm. interossei und lumbri- cales sowie bindegewebige Faserstrukturen. Die palmaren und dorsalen Mm. interossei entspringen den Zwischenräumen der Metakarpalknochen und ziehen in die Strecksehnenaponeurose, selten auch bis in die Grundgelenkkapseln. Die $\mathrm{Mm}$. lumbricales liegen beugeseitig, entspringen den Sehnen des M. flexor digitorum profundus und sind in das lockere Bindegewebe zwischen Palmaraponeurose, $\mathrm{Mm}$. interossei palmares und Beugesehnen eingebettet. Generell führt die intrinsische Muskulatur vorwiegend zur Streckung in den Mittel- und Endgelenken der Finger, die extrinsische Streckmuskulatur dagegen in den Grundgelenken.

Der extrinsische Streckapparat der Finger hat 3 verschiedene Insertionsstellen. Proximal auf Höhe der Mittelhandknochenköpfchen, an der Basis der Mittelphalanx (Mittelzügelansatz) und eine 3. befindet sich weiter distal an der Basis der Endglieder (Ansatz Pars terminalis des lateralen Streckapparats). Auf Höhe der Mittelhandknochen werden die Sehnen seitlich durch Bänder, den Connexus intertendinei (auch: Juncturae tendineae) verbunden. Die Strecksehnenhaube („Extensorhood“) zentriert die Sehnen und verhindert eine Hyperextension. Anschließend verläuft der zentrale Anteil der Sehne als Pars medialis weiter in der Längsachse des Fingers und inseriert am distalen Grundglied und der Basis des Mittelglieds, wo er gemeinsam mit Fasern des Pars lateralis den Tractus intermedius bildet. Die Fasern des Pars lateralis vereinigen sich in Höhe des Grundgliedköpfchens mit den sehnigen Ansätzen der Mm. interossei und Mm. lumbricalis und bilden zusammen den Tractus lateralis. Der radiale und ulnare Tractus lateralis vereinigen sich dann weiter distal zum Pars terminalis und setzen an der Basis des Endglieds an. Diese komplexe anatomische Bauweise ermöglicht, dass die extrinsischen und intrinsischen Muskeln ihre Wirkung auf alle 3 Fingergelenke ausüben (Abb. 1). 


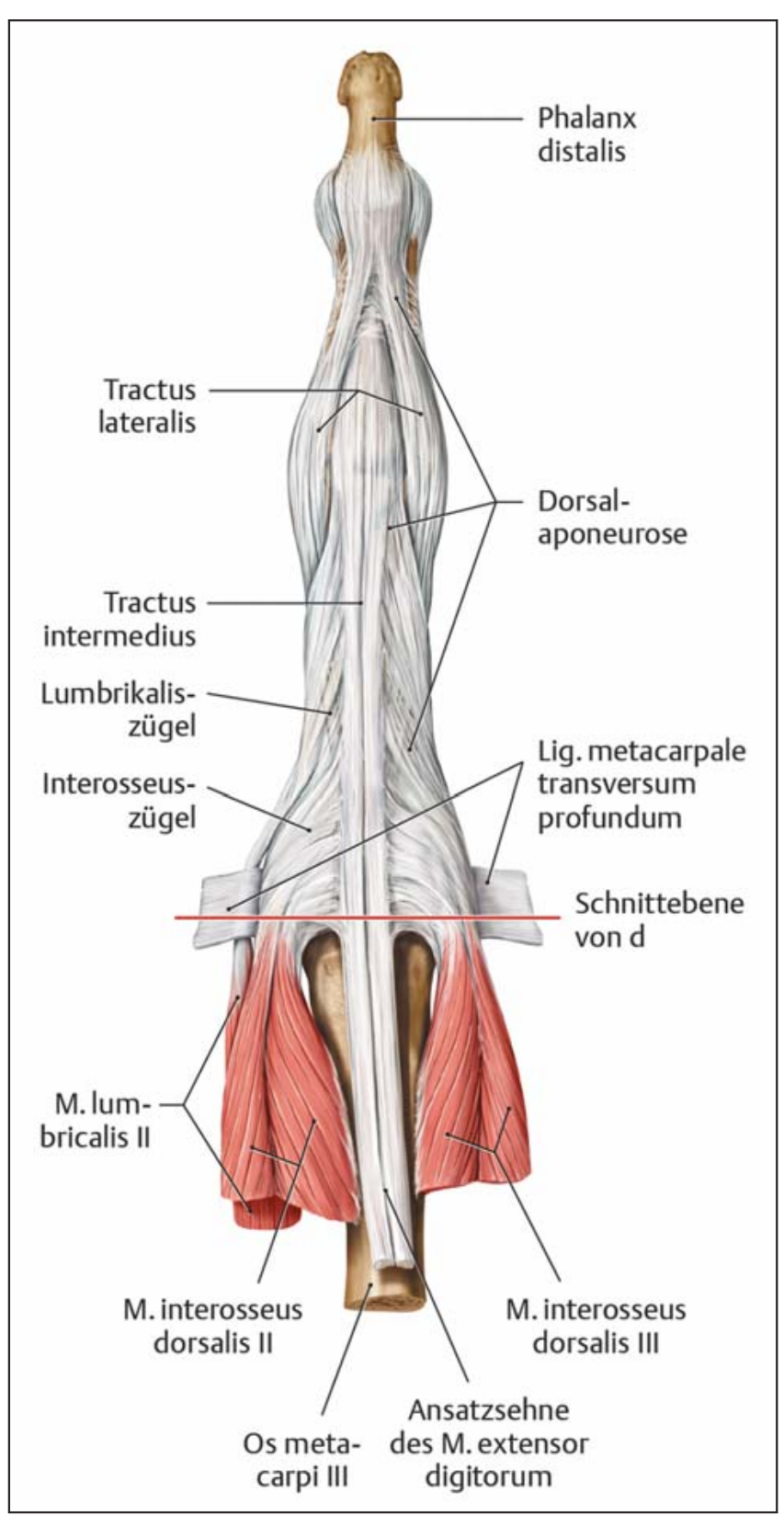

Abb. 1 Anatomischer Überblick der Strecksehnen am Finger. Schünke M, Schulte E, Schumacher U. Prometheus. LernAtlas der Anatomie. Allgemeine Anatomie und Bewegunssystem. Illustrationen von M. Voll und K. Wesker. 3. Aufl. Stuttgart: Thieme; 2011.

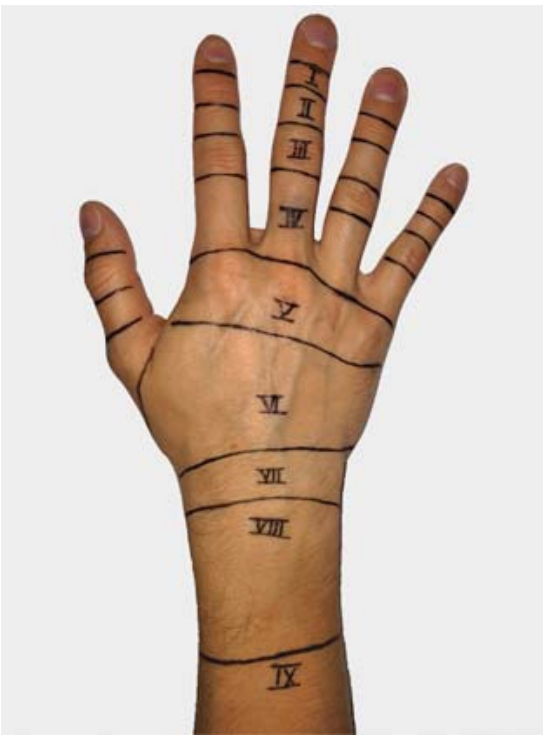

Abb. 2 Zoneneinteilung für Strecksehnenverletzungen der Finger, der Hand und des Unterarms.

Tab. 1 Zonen der Finger, der Hand und des Unterarms.

\begin{tabular}{ll}
\hline Zone 1 & Endgelenk \\
\hline Zone 2 & Mittelglied \\
\hline Zone 3 & Mittelgelenk \\
\hline Zone 4 & Grundglied \\
\hline Zone 5 & Grundgelenk \\
\hline Zone 6 & Mittelhand \\
\hline Zone 7 & Handgelenk \\
\hline Zone 8 & distaler Unterarm \\
\hline Zone 9 & proximaler Unterarm
\end{tabular}

Sehnendurchtrennung die plötzliche gewaltsame Flexion oder eine Luxation nach palmar (Abb. 3b). Meist ist auf dieser Höhe der zentrale Anteil der Strecksehne betroffen und Verletzungen führen dann innerhalb von 10-21 Tagen nach dem initialen Trauma zu einer sog. Knopflochdeformität.

Verletzte Strecksehnen in Zone 4 (Grundglied) sind meist begleitet von offenen Wunden und Begleitfrakturen (Abb. 3c), sind aber häufig nur partiell eingerissen. Eine Durchtrennung des zentralen Anteils kann wiederum zu einer Knopflochdeformität führen.

Strecksehnenverletzungen der Zone 1 (Endgelenk) führen zu einem herunterhängenden Endglied (Abb. $3 a$ ). Bei dieser Deformität spricht man auch von
Verletzungen der Zone 2 (Mittelglied) sind häufig mit Riss- oder Quetschwunden assoziiert und resultieren auch in einem sog. Hammerfinger.

einem Hammerfinger. Neben einer offenen Verletzung spielt hier insbesondere bei gestrecktem Finger eine Rolle. Unbehandelt können der resultierende Spannungsverlust der palmaren Platte am proximalen Interphalangealgelenk und die folgliche Imbalance des Streckapparats $\mathrm{zu}$ einer Schwanenhalsdeformität führen.

Typische Unfallmechanismen in Zone 3 (Mittelgelenk) sind neben der offenen 


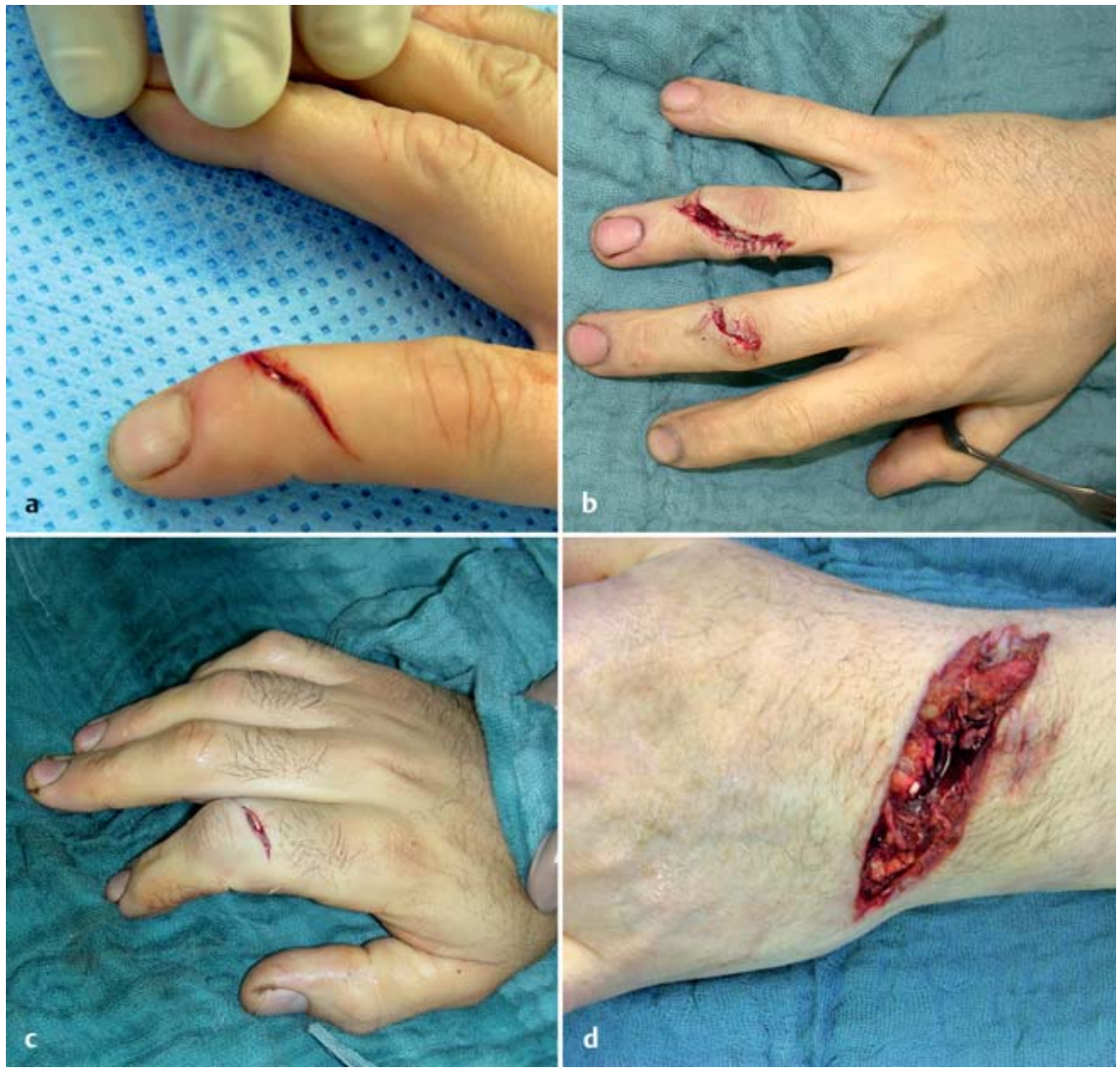

Abb. 3 a bis d Verletzungsmuster von Strecksehnenverletzungen: a Schnittverletzung mit Strecksehnenbeteiligung über dem Endglied (Zone 1). b Riss-/Quetschverletzung über den Mittelgelenken (Zone 3). c Offene Schnittwunde mit verletzter Strecksehne über dem Grundglied in Zone 4. d Komplexe Quetschverletzung am Handgelenk (Zone 7).

Kleinfinger, da diese mit zusätzlichen Strecksehnen ausgestattet sind - dem Extensor indicis proprius und Extensor digiti minimi - sodass die Streckfunktion trotz Sehnendurchtrennung des EDC (Extensor digitorum communis) erhalten ist.

Die Diagnosestellung einer Strecksehnenverletzung in Zone 6 (Mittelhand) ist durch die Connexus intertendinae deutlich erschwert.

Bei Strecksehnenverletzungen proximal der bindegewebigen Faserzüge des Connexus intertendinei zwischen den Fingerstrecksehnen distal des Retinaculum extensorum kann immer noch eine Streckfunktion erhalten bleiben. Sogar bei einer $90 \%$ igen Durchtrennung einer Strecksehne kann die Funktion eines Fingers gegen die Schwerkraft noch erhalten sein.

Strecksehnenverletzungen am Handgelenk in Zone 7 sind normalerweise mit offenen Schnittwunden (Abb.3d) oder distalen Radiusfrakturen mit geschlossenen Rupturen insbesondere der langen Daumenstreckersehne assoziiert. Die häufige Mitbeteiligung des Retinaculum extensorum birgt das Risiko von Adhäsionen postoperativ [4]. Deshalb sollte bei der operativen Versorgung von Strecksehnenverletzungen in dieser Zone das Retinaculum extensorum eröffnet und durch eine Z-Plastik erweitert werden.

In den Zonen 8 und 9 (distaler und proximaler Unterarm) finden sich Verletzungen oft auch an den muskulotendinösen Übergängen oder mit Beteiligung der Muskelbäuche.

\section{Operative Versorgung von Strecksehnendefektverletzungen}

Die Versorgung von Sehnendefektverletzungen bedarf meist komplexer mehrzeitiger Eingriffe und stellt eine besondere Herausforderung für den behandelnden Chirurgen dar. Operative Eingriffe bei komplexen Handverletzungen erfolgen meist in Rückenlage des Patienten, Plexusanästhesie und Oberarmblutleere sowie unter Lupenvergrößerung [5]. Zunächst beginnt die Operation mit einem ausgiebigen Débridement und Spülen der Wunde [6].
Die Sehnen sollten immer zart behandelt werden, übermäßige Druckkräfte oder Punktionen mit einer Pinzette und groben Nadeln müssen vermieden werden, um Adhäsionen und Narbenbildungen vorzubeugen.

\section{Sehnenumlagerung}

Die technisch einfachste Methode zur Rekonstruktion von Strecksehnendefektverletzungen sind sog. Umkehrplastiken wie die Snow-Plastik [7], mit denen kleine zentrale Sehnendefektverletzungen rekonstruiert werden können. Dabei wird ein ca. $1 \mathrm{~cm}$ langer zungenförmiger Sehnenstreifen aus einem proximalen Sehnenstumpf zentral aus der Pars medialis umschnitten und distal gestielt gelassen. Zur Defektdeckung wird der Sehnenzügel dann um $180^{\circ}$ rotiert und als Brücke nach distal umgeschlagen. Dann wird der Sehnenstreifen mit dem distalen Sehnenstumpf vernäht oder transossär mittels Knochenanker refixiert. Die Umschlagstelle wird schließlich mit einzelnen Nähten gesichert und die Entnahmestelle mit einer fortlaufenden Naht verschlossen (Abb.4). Zudem kann ggf. die temporäre Transfixation des Gelenks mittels eines Kirschner-Drahtes notwendig sein. Eine Schienenruhigstellung ist im Anschluss für 4-6 Wochen indiziert.

\section{Sehnenkoppelung}

Neben Umkehrplastiken spielt der heterodigitale Sehnentransfer zum Ersatz einer fehlenden Streckfunktion eine wichtige Rolle. Die Sehne des M. extensor indicis proprius (EI) hat für den Zeigefinger eine redundante Funktion mit der EDC-Sehne und ist somit entbehrlich. Allerdings sollte der Chirurg sich vor einer Sehnenübertragung v.a. bei Patienten mit rheumatoider Arthritis sicher sein, dass die EDC-II-Sehne noch normal funktionsfähig ist. In diesem Sinne wird die EPL-Funktion (Extensor pollis longus) üblicherweise durch einen EI-Transfer ersetzt und zeigt sogar vergleichbare postoperative Ergebnisse mit einer freien Sehnentransplantation wie bspw. mit der Sehne des M. palmaris longus [8]. Die EI-Sehne liegt ulnar der EDCSehne. Sie wird zunächst freipräpariert, distal scharf durchtrennt, mobilisiert und zu den distalen Sehnenstümpfen meist zum distalen Anteil der EPL verlagert. Bei ausreichender Länge erfolgt die Sehnennaht nach Pulvertaft, ansonsten End-zu-End mit U-Nähten (Abb.5). Bei der Pulvertaft-Naht wird das Ende der dünnen Seite mittels eines Skalpells 


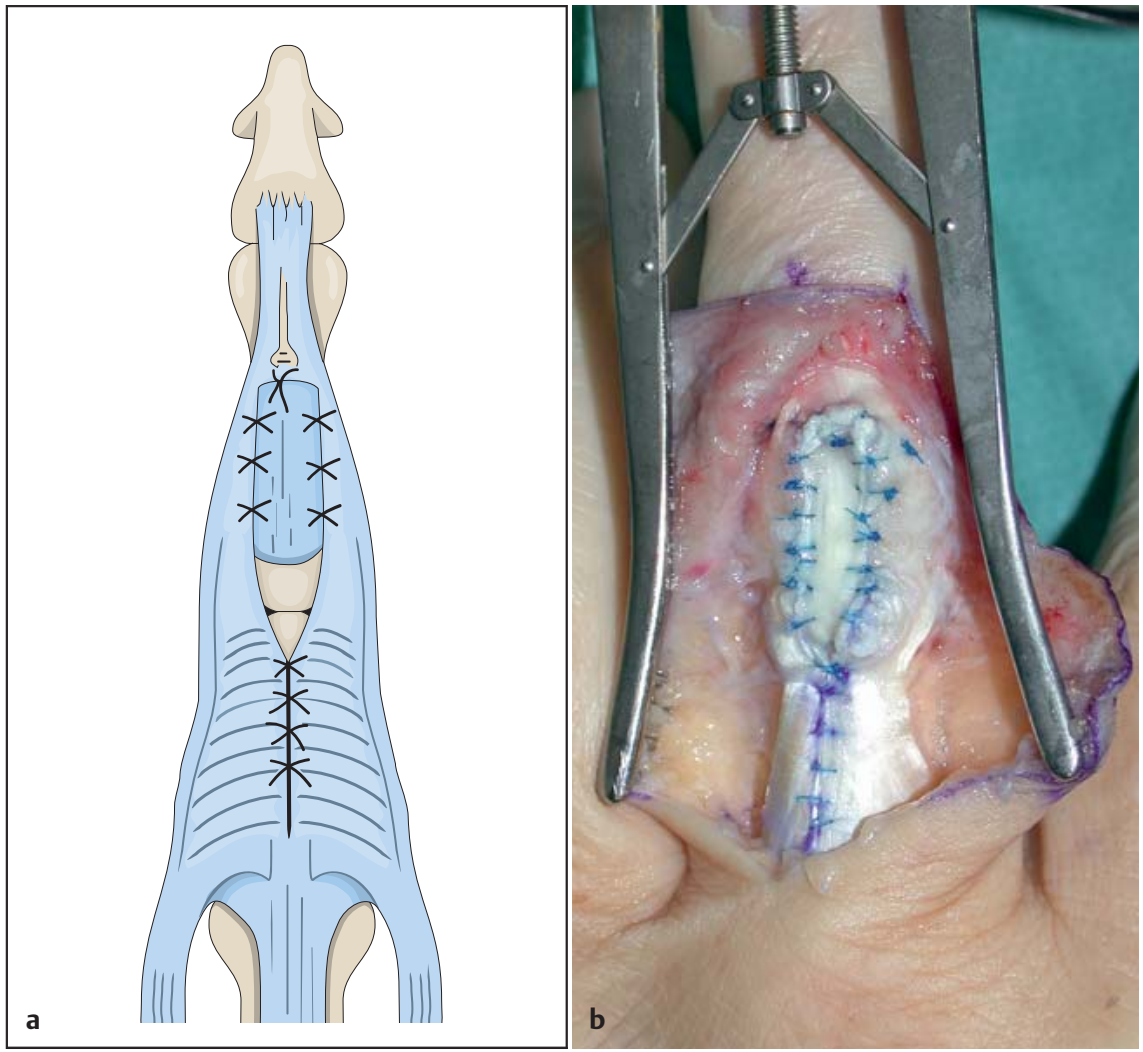

Abb. 4 Schematische und klinische Darstellung der Umkehrplastik nach Snow zur Rekonstruktion kleiner zentraler Sehnendefektverletzungen.

oder einer Durchflechtungszange und einer feinen Klemme mehrmals durch die kräftigere Sehne durchgezogen. Dann erfolgt die Längsspaltung des freien Endes der kräftigen Sehne zur Aufnahme der dünneren Sehne und beide Enden werden miteinander vernäht. Durch Längsspaltung der Indicissehne ist sogar die Versorgung mehrerer Fingerstrecksehnen möglich. Für die Langfingerstrecker dagegen reicht meist ein Seit-zuSeit-Transfer mit einer benachbarten Strecksehne wie die Übertragung einer EDC-Sehne und bietet damit bereits eine ausreichende Funktion. Postoperativ sollte eine gesamte Ruhigstellungszeit von 4 Wochen nicht überschritten werden und eine Frühmobilisation mittels dynamischer Schiene erfolgen.

\section{Sehnentransplantation}

Freie Sehnentransplantationen zur Defektüberbrückung der Strecksehnen erfordern eine gute elastische und verschiebliche Weichgewebsdecke und ein infektfreies Sehnenlager. Als Transplantat eignet sich besonders die Sehne des M. palmaris longus (PL). Dieser oberflächlichste Muskel aller Handgelenksbeuger entspringt am Epicondylus medialis des Humerus sowie an der Fascia antebrachii und inseriert am Lig. carpi transversum und an der Palmaraponeurose in der Handinnenfläche. Durch Opposition des Daumens bei gleichzeitiger Beugung des Handgelenks gegen Widerstand wird die Sehne mittig des Handgelenks sehr prominent sichtbar. Diese präoperative klinische Untersuchung ist unerlässlich, da die akzessorische Sehne in etwa $20 \%$ der Fälle ein- oder beidseitig fehlt. Zur Entnahme des PL-Sehnentransplantats wird direkt unter der Haut und oberhalb der Unterarmfaszie die PLSehne freigelegt, mobilisiert und distal durchtrennt. Die Entnahme erfolgt dann mit einem Sehnenstripper. Dazu wird das proximale Ende des PL an einem Haltefaden befestigt, durch den Stripperring eingeführt und der Stripper dann unter Zug der Sehne proximalwärts unter drehenden Bewegungen vorgeschoben. Proximal wird die Sehne durch den Ring im Bereich des Muskelbauchs abgetrennt und kann schließlich hervorgezogen werden (Abb. 6). Nach der langstreckigen PL-Entnahme kann das Transplantat zwischen die verletzten Sehnenstümpfe zur Defektüberbrückung üblicherweise nach Pulvertaft oder mit U-Nähten eingenäht werden. Diese Nahttechnik erweist sich als äußerst stabil und erlaubt eine frühzeitige Mobilisation. Ein seltener genutztes Transplantat bei Defektverletzungen der Strecksehnen ist die Sehne des M. plantaris longus. Die Sehne liegt zwischen Innenknöchel und Achillessehne. Aber Vorsicht, oft fehlt auch die Plantarissehne, wenn der M. palmaris longus genetisch nicht angelegt ist. Auch hier erfolgt die Entnahme der Sehne nach vorangegangener Identifikation und Freilegung mit einem langen Sehnenstripper.

\section{Vaskularisierte Sehnentransplantation}

Die Rekonstruktion von Strecksehnendefektverletzungen, v.a. bei gleichzeitigem Vorliegen von Hautweichteildefekten
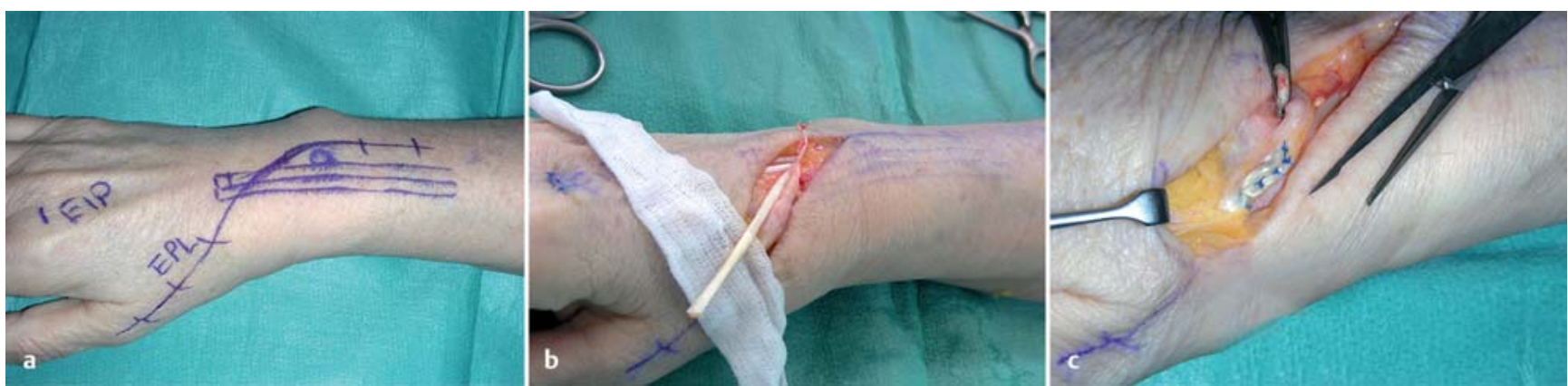

Abb. $\mathbf{5}$ a bis c Extensor-indicis-proprius-Transfer zur Rekonstruktion der Extensor-pollicis-longus-Sehne. a Anzeichnung zur OP-Planung. $\mathbf{b}$ Distal abgesetzte El-Sehne auf der Kompresse und radial die durchtrennte EPL-Sehne. c Sehnennaht nach Pulvertaft mithilfe der Pulvertaft-Klemme rechts im Bild. 


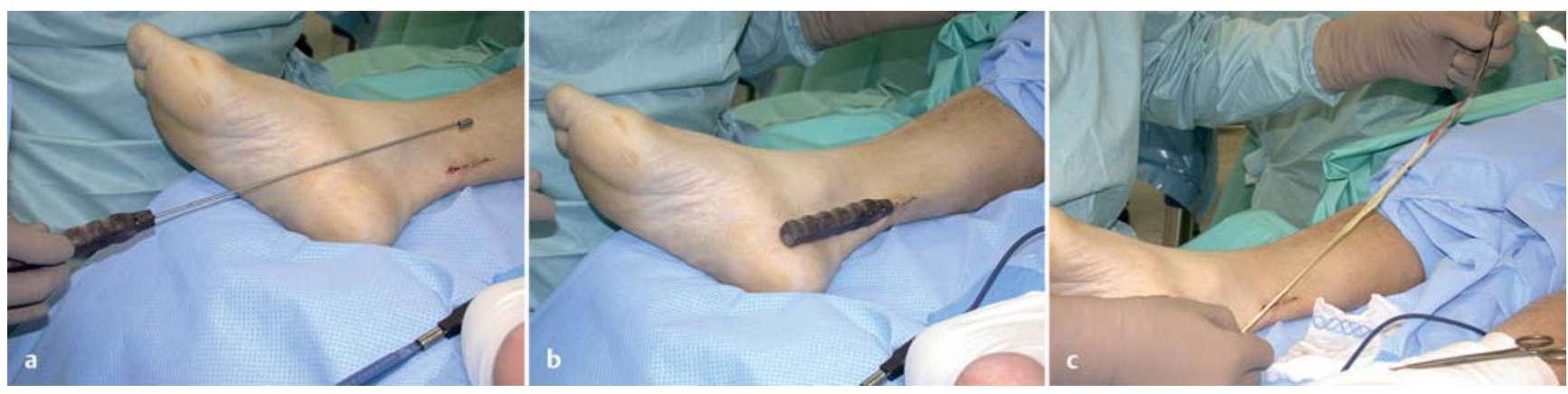

Abb. $\mathbf{6 a}$ bis c Entnahme der Sehne des M. plantaris longus zur Sehnentransplantation mit einem Sehnenstripper (a-c).
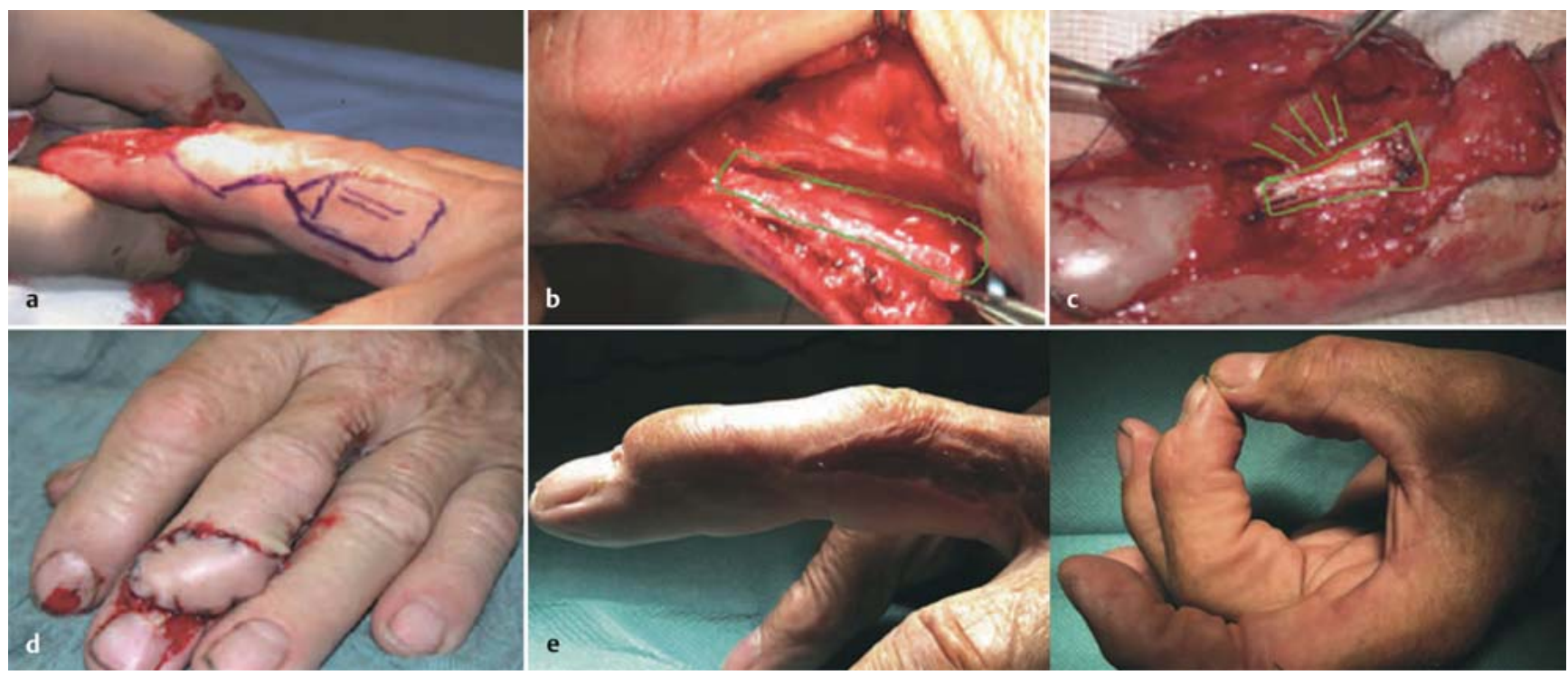

Abb. 7a bis e Vaskularisierte Sehnentransplantation mit homodigitalem Insellappen zur Defektdeckung über dem Endgelenk. a Anzeichnen des Lappendesigns und des darunterliegenden Sehnenanteils. b Präparation und Mobilisierung der vaskularisierten Sehne eingebettet in den Insellappen. c Rotation des gefäßgestielten Lappens nach distal auf den Defekt und Sehnennaht. d Unmittelbar postoperatives Ergebnis und e 6 Monate nach OP.

nach komplexen Verletzungen an den Fingern, erfordert eine besondere handchirurgische Expertise. Elegante Möglichkeiten zur funktionellen und ästhetischen Rekonstruktion von komplexen dorsalen Fingerdefektverletzungen bieten die vaskularisierten Sehnentransplantate in Kombination mit Insellappenplastiken. Dabei wird ein vaskularisiertes Sehnentransplantat integriert in einem Hautlappen gehoben, um den Verlust von Hautgewebe und Strecksehne im Defektbereich einzeitig wiederherzustellen. Beispiele dafür sind die homodigitalen oder heterodigitalen Insellappen, die z.B. Sehnenanteile vom Tractus lateralis beinhalten. Hier sollte zunächst präoperativ eine sorgfältige dopplersonografische Beurteilung der Durchblutung der Finger erfolgen, um Perfusionskomplikationen am Hebedefekt zu verhindern. Je nach Befund und Durchblutungssituation kann hier ein homodigitaler oder heterodigitaler Insellappen mit darunterliegendem Sehnen- anteil für die Deckung geplant werden. Intraoperativ wird nach Bestimmung des Lappendesigns zunächst das GefäßNerven-Bündel am distalen oder proximalen Lappen identifiziert. Die Arterie und die dazugehörigen Begleitvenen werden nun vorsichtig vom Nerv separiert. Dann wird der vaskularisierte Teil des Tractus lateralis gemeinsam mit dem Lappen gehoben und die Gefäße bis zu einer ausreichenden Länge präpariert. Folglich kann das kombinierte Lappen-Sehnen-Transplantat gestielt auf die Empfängerstelle desselben (homodigital, Abb. 7) oder benachbarten (heterodigital) Fingers transferiert werden [9]. Der verbliebene Defekt an der Spenderregion kann je nach Ausmaß direkt oder mit einem Vollhauttransplantat bspw. vom Unterarm verschlossen werden. Eine vorübergehende Kirschner-Draht-Transfixation z.B. bei gelenkübergreifenden Defekten kann die Ruhigstellung sichern. Die postoperative Ruhigstellung erfolgt in Abhängigkeit des Verletzungsmusters mit einer angepassten funktionellen Schienenbehandlung für mindestens 6 Wochen.

Eine ähnliche Technik zur Behandlung kombinierter Hautweichteil- und Sehnendefektverletzungen ist ein distal gestielter DMCA-Lappen (DMCA: Dorsal Metacarpal Artery) mit inkorporierter EI-Sehne zur Rekonstruktion von Defektverletzungen streckseitig über dem Mittel- und Endgelenk insbesondere am Zeigefinger [10]. Hierzu wird ein distal gestielter Lappen aus dem 2. intermetakarpalen Raum als fasziotendokutaner Insellappen mit Einbindung der darunterliegenden EI-Sehne verwendet. Die EI-Sehne wird in entsprechender Länge reseziert, mit dem Lappen mobilisiert und gestielt auf dem Defekt nach distal transferiert (Abb. 8).

Ziel dieser Methoden ist der erfolgreiche Erhalt der Fingerlänge, Beweglichkeit und des Feingefühls und weitere Finger- 

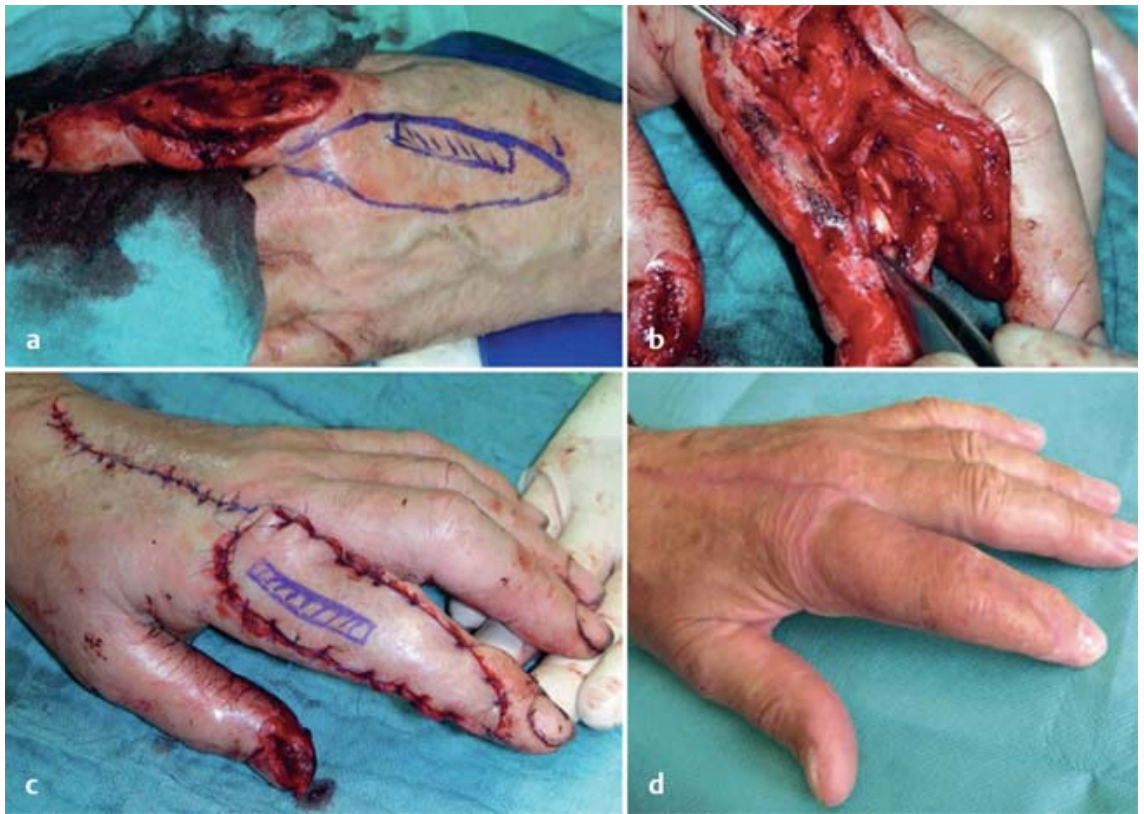

Abb. 8 a bis d DMCA-Lappen (DMCA: Dorsal Metacarpal Artery) mit inkorporierter El-Sehne zur Rekonstruktion einer Defektverletzung streckseitig über dem Zeigefingermittelgelenk. a Anzeichnen des Lappendesigns und des darunterliegenden Sehnenanteils. b Präparation und Mobilisierung der vaskularisierten Sehne eingebettet in dem DMCA-Lappen auf die Defektverletzung am Zeigefinger. c Unmittelbar postoperatives Ergebnis und d 3 Monate nach OP.

deformitäten, Arthrodesen oder Amputationen insbesondere nach komplexen Verletzungen des Streckapparats zu verhindern. Zudem sichert nur ein intensives postoperatives Behandlungsmanagement mit adäquater Ruhigstellung und funktioneller Nachbehandlung den Erfolg der Behandlung.

\section{Schlussfolgerung}

Zusammenfassend sind die Voraussetzungen für eine adäquate Versorgung von komplexen Strecksehnenverletzungen an den Phalangen gute anatomische Kenntnisse und handchirurgische Erfahrung. Für die Behandlung existieren verschiedene therapeutische Richtlinien in Abhängigkeit von der Lokalisation, den anatomischen Gegebenheiten und dem
Verletzungsmuster. Dabei ist eine individuell angepasste Nachsorge mit ausreichend langer Ruhigstellung und frühfunktioneller Nachbehandlung maßgeblich, um Adhäsionen und Bewegungseinschränkungen der Hand zu vermeiden und den Erfolg der Behandlung zu sichern.

\section{Literatur}

${ }^{1}$ Hanz KR, Saint-Cyr M, Semmler MJ et al. Extensor tendon injuries: acute management and secondary reconstruction. Plast Reconstr Surg 2008; 121: 109e-120e

2 Kleinert HE, Verdan C. Report of the Committee on Tendon Injuries (International Federation of Societies for Surgery of the Hand). J Hand Surg Am 1983; 8: 794-798

3 Verdan C. [Historical development of surgery of the flexor tendons]. Handchirurgie 1981; 13: $181-185$
${ }^{4}$ Tang JB. Tendon injuries across the world: treatment. Injury 2006; 37: 1036-1042

${ }^{5}$ Flegal MC, Fox LK, Kuhlman SM. Principles of anesthesia monitoring and electrocardiogram. J Invest Surg 2009; 22: 316-317

${ }^{6}$ Brzezienski MA, Schneider LH. Extensor tendon injuries at the distal interphalangeal joint. Hand Clin 1995; 11: 373-386

7 Snow JW. A method for reconstruction of the central slip of the extensor tendon of a finger. Plast Reconstr Surg 1976; 57: 455-459

8 Schaller P, Baer W, Carl HD. Extensor indicistransfer compared with palmaris longus transplantation in reconstruction of extensor pollicis longus tendon: a retrospective study. Scand J Plast Reconstr Surg Hand Surg 2007; 41: 33-35

${ }^{9}$ Rahmanian-Schwarz A, Schiefer J, Amr A et al. Vascularized tendon incorporated in reverse homodigital and heterodigital island flaps for the reconstruction of dorsal digital defects. Microsurgery 2012; 32: 178-182

10 Schiefer JL, Schaller HE, Rahmanian-Schwarz A. Dorsal metacarpal artery flaps with extensor indices tendons for reconstruction of digital defects. J Invest Surg 2012; 25: 340-343

\section{Dr. med. Wiebke Petersen \\ Assistenzärztin}

Univ.-Prof. Dr. med. Hans-Eberhard Schaller

Ärztlicher Direktor

Klinik für Hand-, Plastische, Rekonstruktive und Verbrennungschirurgie Eberhard-Karls-Universität Tübingen, BG Unfallklinik

Schnarrenbergstraße 95

72076 Tübingen

\section{Prof. Dr. med. Afshin Rahmanian-Schwarz Ärztlicher Direktor}

Klinik für Plastische und Handchirurgie, Ästhetische und Rekonstruktive Chirurgie Universität Witten/Herdecke, Helios Klinikum Wuppertal

Heusnerstraße 40 42283 Wuppertal

Afshin.Rahmanian-Schwarz@ helios-kliniken.de 